\title{
Application of Lanczos and Schur vectors in structural dynamics
}

\author{
M. Radeş \\ Universitatea Politehnica Bucureşti, Splaiul Independenţei 313, Bucureşti, Romania \\ E-mail: rades@form.resist.pub.ro
}

Received 2007

Revised 2007

\begin{abstract}
The paper explains the advantages of using Lanczos/Schur vectors when performing dynamic analyses of very large models: faster computation of low spectrum eigenproperties, reduced computer storage requirements, increased robustness of test-analysis models, and sensor location by the effective independence method without solving large size eigenproblems.
\end{abstract}

Keywords: Schur vectors, Lanczos vectors, Schur TAM, Lanczos TAM

\section{Introduction}

Subspace iteration methods, based on Rayleigh-Ritz approximations, are used in the solution of large eigenvalue problems arising in the dynamic analysis of structures described by very large sparse or structured matrices [1]. Eigenvalues are the diagonal entries of an upper triangular Schur form of the projected system matrix, and eigenvectors can be computed as linear combinations of Schur vectors [2]. Eigenvectors can also be expressed as linear combinations of Lanczos vectors. The projection of the operating matrix onto the subspace of Lanczos vectors yields a tridiagonal matrix whose eigenvalues are used to approximate some of the eigenvalues of the data matrix. Excellent approximations to a few eigenvalues can be obtained after a number of iterations significantly smaller than the system order $[3,4]$. The paper shows how Schur and Lanczos vectors can be used in model reduction and correlation applications without solving large scale eigenvalue problems.

\section{Structural dynamics eigenproblem}

Modal properties such as natural frequencies and mode shape vectors of conservative structural systems are obtained solving the generalized symmetric eigenproblem

$$
(\mathbf{K}-\lambda \mathbf{M}) \mathbf{x}=0,
$$

where the stiffness and mass matrices, $\mathbf{K}$ and $\mathbf{M}$, are $n$ by $n$ real and symmetric, $\lambda$ are real eigenvalues and $\mathbf{x}$ are real eigenvectors. The usual approach is to transform Eq. (1) into an ordinary eigenvalue problem

$$
\mathbf{A} \mathbf{x}=\theta \mathbf{x} .
$$

This is done considering a shift $\sigma$ and writing the problem Eq. (1) in the form $(\mathbf{K}-\sigma \mathbf{M})^{-1} \mathbf{M} \mathbf{x}=(\lambda-\sigma)^{-1} \mathbf{x}$. Denoting

$$
\mathbf{A}=(\mathbf{K}-\sigma \mathbf{M})^{-1} \mathbf{M}, \quad \theta=(\lambda-\sigma)^{-1},
$$




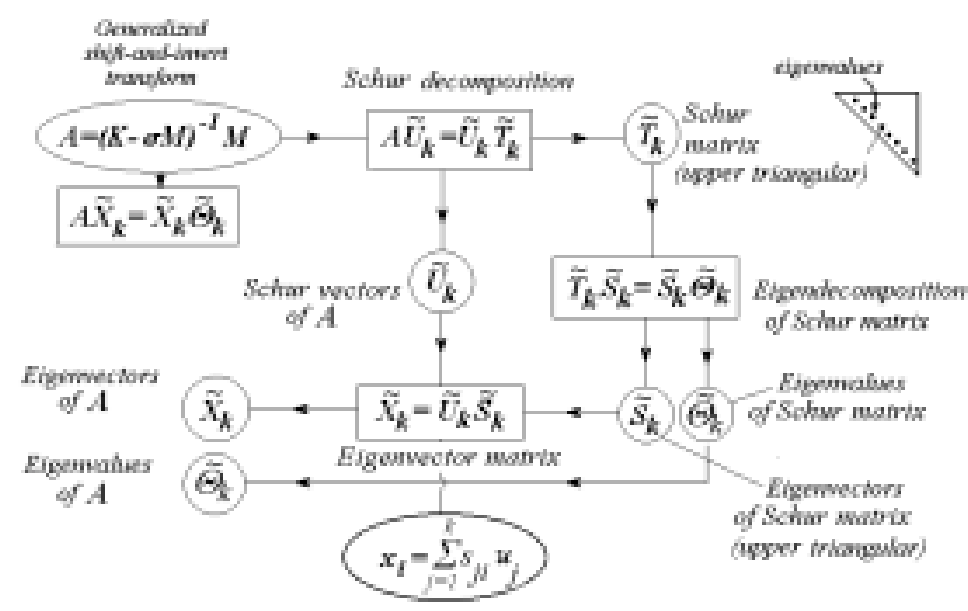

Fig. 1. Schematic of eigensolution using Schur vectors.

we obtain Eq. (2) where the non-symmetric matrix $\mathbf{A}$ is called the generalized shift-and-invert transform [1]. The eigenvalues of the pencil $(\mathbf{K}, \mathbf{M})$ that are near $\sigma$ become large, so that they will tend to be found first. The matrix $\mathbf{A}$ is never constructed, and an LU factorization is used with a matrix-vector product.

The goal of dense eigenvalue computations is the systematic reduction of matrices to simpler form by similarity transformations. The common approach used in structural dynamics is the reduction to diagonal form. Denoting

$$
\tilde{\mathbf{X}}_{k}=\left(\mathbf{x}_{1}, \ldots, \mathbf{x}_{k}\right), \quad \tilde{\Theta}_{k}=\operatorname{diag}\left(\theta_{1}, \ldots, \theta_{k}\right),
$$

the individual relations $\mathbf{A x}_{k}=\theta_{k} \mathbf{x}_{k}$ can be combined in

$$
\mathbf{A} \tilde{\mathbf{X}}_{k}=\tilde{\mathbf{X}}_{k} \tilde{\Theta}_{k},
$$

where a tilde over letter denotes quantities not obtained by projection on a subspace.

An alternative approach is to use the QR algorithm to perform a Schur decomposition (Fig. 1). In this case, the working matrix is reduced to upper triangular form by orthogonal similarity transformations. This is computationally more stable and less expensive than the reduction to diagonal form.

\section{Schur vectors}

For a selected set of $k$ (e.g.: largest) eigenvalues of $\mathbf{A}$, there is a partial Schur decomposition

$$
\mathbf{A} \tilde{\mathbf{U}}_{k}=\tilde{\mathbf{U}}_{k} \tilde{\mathbf{T}}_{k},
$$

where $\tilde{\mathbf{T}}_{k}$ is upper triangular. $\tilde{\mathbf{U}}_{k}$ is orthogonal and its columns are Schur vectors of $\mathbf{A}$. The diagonal elements of $\tilde{\mathbf{T}}_{k}$ are eigenvalues of $\mathbf{A}$. By appropriate choice of $\tilde{\mathbf{U}}_{k}$ they may be made to appear in any specified order. The Schur matrix $\tilde{\mathbf{T}}_{k}$ has an eigendecomposition

$$
\tilde{\mathbf{T}}_{k} \tilde{\mathbf{S}}_{k}=\tilde{\mathbf{S}}_{k} \tilde{\Theta}_{k},
$$

where $\tilde{\mathbf{S}}_{k}$ is the upper triangular matrix of the eigenvectors of $\tilde{\mathbf{T}}_{k}$, and $\tilde{\Theta}_{k}$ is the diagonal matrix of the eigenvalues from Eq. (4). It turns out that the eigenvector matrix $\tilde{\mathbf{X}}_{k}$ is given by

$$
\tilde{\mathbf{X}}_{k}=\tilde{\mathbf{U}}_{k} \tilde{\mathbf{S}}_{k},
$$

so that the eigenvectors of $\mathbf{A}$ are linear combinations of the orthogonal Schur vectors corresponding to the selected eigenvalues

$$
\mathbf{x}_{i}=\sum_{j=1}^{k} s_{j i} \mathbf{u}_{j} .
$$


If the Schur vectors are $M$-orthonormal, then $\tilde{\mathbf{U}}_{k}^{T} \mathbf{M} \tilde{\mathbf{U}}_{k}=\mathbf{I}_{k}$. Because matrices $\mathbf{K}$ and $\mathbf{M}$ are symmetric, the product MA is symmetric. Then $\tilde{\mathbf{U}}_{k}^{T} \mathbf{M A} \tilde{\mathbf{U}}_{k}=\tilde{\mathbf{T}}_{k}$ is a Schur form of MA, $\tilde{\mathbf{T}}_{k}^{T}=\tilde{\mathbf{T}}_{k}$, so that $\tilde{\mathbf{T}}_{k}$ itself is symmetric, hence is diagonal. Its elements are eigenvalues of $\mathbf{A}$ and the Schur vectors are eigenvectors of $\mathbf{A}$.

For large order systems, it is better to solve the generalized Hermitian eigenproblem Eq. (1) without transformation to a standard eigenproblem. For stability reasons, it is more appropriate to work with orthogonal transformations and to compute Schur vectors for the pencil $\mathbf{A}-\lambda \mathbf{B}$ rather than eigenvectors.

A partial generalized Schur form of dimension $k$ for the matrix pair $(\mathbf{K}, \mathbf{M})$ is the decomposition

$$
\mathbf{K} \tilde{\mathbf{Z}}_{k}=\tilde{\mathbf{Q}}_{k} \tilde{\mathbf{T}}_{k}^{K}, \quad \mathbf{M} \tilde{\mathbf{Z}}_{k}=\tilde{\mathbf{Q}}_{k} \tilde{\mathbf{T}}_{k}^{M},
$$

where $\tilde{\mathbf{Z}}_{k}$ and $\tilde{\mathbf{Q}}_{k}$ are orthonormal $n$ by $k$ matrices, and $\tilde{\mathbf{T}}_{k}^{K}$ and $\tilde{\mathbf{T}}_{k}^{M}$ are upper triangular $k$ by $k$ matrices. The columns of $\tilde{\mathbf{Z}}_{k}$ (and $\tilde{\mathbf{Q}}_{k}$ ) are referred to as generalized Schur vectors. In our applications they have been computed using the JDQR algorithm [2], a subspace iteration variant of the QR algorithm. Eigenvalues are computed from the ratio of the diagonals of the triangular forms.

\section{Subspace iteration methods}

The eigenproblem of large order structural models is solved using the Rayleigh-Ritz method. This method is used for extracting an approximate low-dimensional eigenspace from a larger subspace. It is possible to construct $k$ approximate eigenvectors of $\mathbf{A}, \mathbf{X}_{k}=\left(\mathbf{x}_{1}, \mathbf{x}_{2}, \ldots, \mathbf{x}_{k}\right)$, as linear combinations of some trial vectors $\mathbf{V}_{m}=$ $\left(v_{1}, v_{2}, \ldots, v_{m}\right)$ :

$$
\mathbf{X}_{k}=\mathbf{V}_{m} \mathbf{Y}_{k}, \quad \mathbf{x}_{i}=\sum_{j=1}^{m} y_{j i} v_{j}
$$

where $\mathbf{Y}_{k}=\left(\mathbf{y}_{1}, \mathbf{y}_{2}, \ldots, \mathbf{y}_{k}\right)$ and $k<<n$. In the reference [3], $m=2 k$.

Any pair $\left(\mathbf{x}_{i}, \theta_{i}\right)$, that satisfies the $M$-orthogonality condition for residuals

$$
\mathbf{V}_{m}^{T} \mathbf{M}\left(\mathbf{A} \mathbf{x}_{i}-\theta_{i} \mathbf{x}_{i}\right)=0
$$

is called a Ritz pair. For $k$ eigenpairs, Eq. (11) can be written

$$
\mathbf{V}_{m}^{T} \mathbf{M}\left(\mathbf{A} \mathbf{X}_{k}-\mathbf{X}_{k} \Theta_{k}\right)=0
$$

where $\Theta_{k}=\operatorname{diag}\left(\theta_{1}, \theta_{2}, \ldots, \theta_{k}\right)$. Substituting Eq. (10) into Eq. (12), one obtains the reduced eigensystem

$$
\mathbf{H}_{m} \mathbf{Y}_{k}-\mathbf{Y}_{k} \Theta_{k}=0,
$$

where

$$
\mathbf{H}_{m}=\mathbf{V}_{m}^{T} \mathbf{M A ~} \mathbf{V}_{m}
$$

has the form of a Rayleigh quotient matrix, and $\mathbf{V}_{m}^{T} \mathbf{M} \mathbf{V}_{m}=\mathbf{I}_{m}$.

The columns of $\mathbf{X}_{k}$ are called the Ritz vectors, and the elements of $\Theta_{k}$ are the Ritz values. The columns of $\mathbf{Y}_{k}$ are referred to as primitive Ritz vectors. The Rayleigh-Ritz approximation Eq. (10) allows constructing eigenpairs $\left(\mathbf{x}_{i}, \theta_{i}\right)$ of the large matrix $\mathbf{A}$, from the eigenpairs $\left(\mathbf{y}_{i}, \theta_{i}\right)$ of the small matrix $\mathbf{H}_{m}$. Figure 2 shows the relationships between the quantities involved in computation.

\section{Lanczos vectors}

Krylov subspace methods encompass three main steps: a) matrix preparation, b) subspace generation, and c) eigenvalue approximation. If $m<<n$, the eigensystem of $\mathbf{H}_{m}$ can be computed by conventional (dense) means. An appropriate form of $\mathbf{H}_{m}$ for the QR iteration is the Hessenberg form. This can be obtained using the Arnoldi factorization [1], i.e. selecting the columns of $\mathbf{V}_{m}$ as orthonormal Arnoldi vectors. 


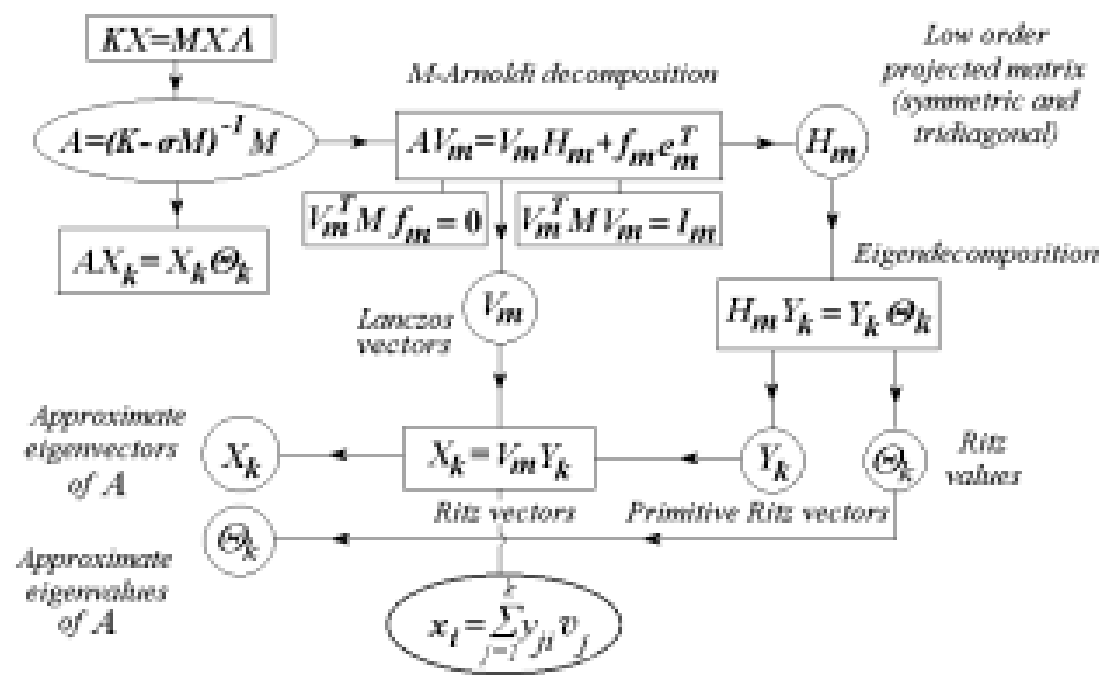

Fig. 2. Schematic of the $M$-Arnoldi process.

Arnoldi vectors are orthogonal Krylov vectors, generated by repeated multiplication of a starting vector with the working matrix and reorthogonalization [2]. The subspace is generated by adding one vector at a time and orthogonalizing. Vectors already converged are locked, fake vectors are purged from the basis, the others are used for further computation in implicit restart.

In our computations we used a modified version of the eigs.m function from the MATLAB5 implementation [3] of the Implicitly Restarted Arnoldi iteration method [4]. The IRA algorithm is used with repeated, carefully chosen restarts, to keep small the storage space. Quantities to be decided are the convergence tolerance $\left\|\mathbf{A} \mathbf{X}_{k}-\mathbf{X}_{k} \Theta_{k}\right\|_{1} /\|\mathbf{A}\|_{1}$, the starting vector, the number of desired eigenpairs, the dimension of the Arnoldi basis necessary to ensure the prescribed accuracy, the stagnation tolerance and the maximum number of Arnoldi iterations. Some details are given in [5]. Using $M$-orthogonal Arnoldi vectors, the matrix Eq. (16) is symmetric (tridiagonal) and the Arnoldi process reduces to the more simple Lanczos process for the symmetric problem.

The result of the $M$-Arnoldi decomposition is a set of Lanczos vectors collected in $\mathbf{V}_{m}$ and the low-order projected matrix $\mathbf{H}_{m}=\mathbf{V}_{m}^{T} \mathbf{M A ~} \mathbf{V}_{m}$, which is symmetric and tridiagonal. One way further is to directly use the eigendecomposition of $\mathbf{H}_{m}$. Its eigenvalues are used to approximate some of the eigenvalues of the data matrix. The eigenvectors of the operating matrix are expressed as linear combinations of the Lanczos vectors, with the multiplying factors as elements of the eigenvectors of $\mathbf{H}_{m}$ (Fig. 2). The power of the method consists in the fact that excellent approximations to a few eigenvalues can be obtained after a number of iterations significantly smaller than the order of the working matrix.

Another general approach (Fig. 3) is to first compute a partial Schur form of $\mathbf{H}_{m}$, then to use its eigendecomposition to obtain approximate eigenvalues of $\mathbf{A}$ [6]. The matrix of primitive Ritz vectors $\mathbf{Y}_{k}$ can be obtained premultiplying the eigenmatrix of $\mathbf{R}_{k}$ by the matrix of Schur vectors of $\mathbf{H}_{m}$. Approximate eigenvectors of $\mathbf{A}$ are then obtained as before using the Lanczos vectors. Alternatively, approximate Schur vectors of $\mathbf{A}$ are first obtained multiplying the matrix of Lanczos vectors by the matrix of Schur vectors of $\mathbf{H}_{m}$. Then, Ritz vectors of $\mathbf{A}$ are obtained multiplying $\mathbf{U}_{k}$ by the eigenmatrix of $\mathbf{R}_{k}$. For the $M$-Arnoldi process the eigendecomposition of $\mathbf{R}_{k}$ is not necessary. It is mentioned here because it is implemented in existing codes.

In the following, Eqs (7), (8) and (10) will be exploited. They show that the Ritz vectors of the working matrix are linear combinations of either the Lanczos vectors or the Schur vectors. Use of these orthogonal vectors instead of the eigenvectors is justified by the fact that they are obtained before eigenvectors and with less computational effort. Their use in sensor location and test-analysis correlation provides comparable accuracy and increased robustness, providing a more suitable subspace for model reduction than the eigenvector subspace. 


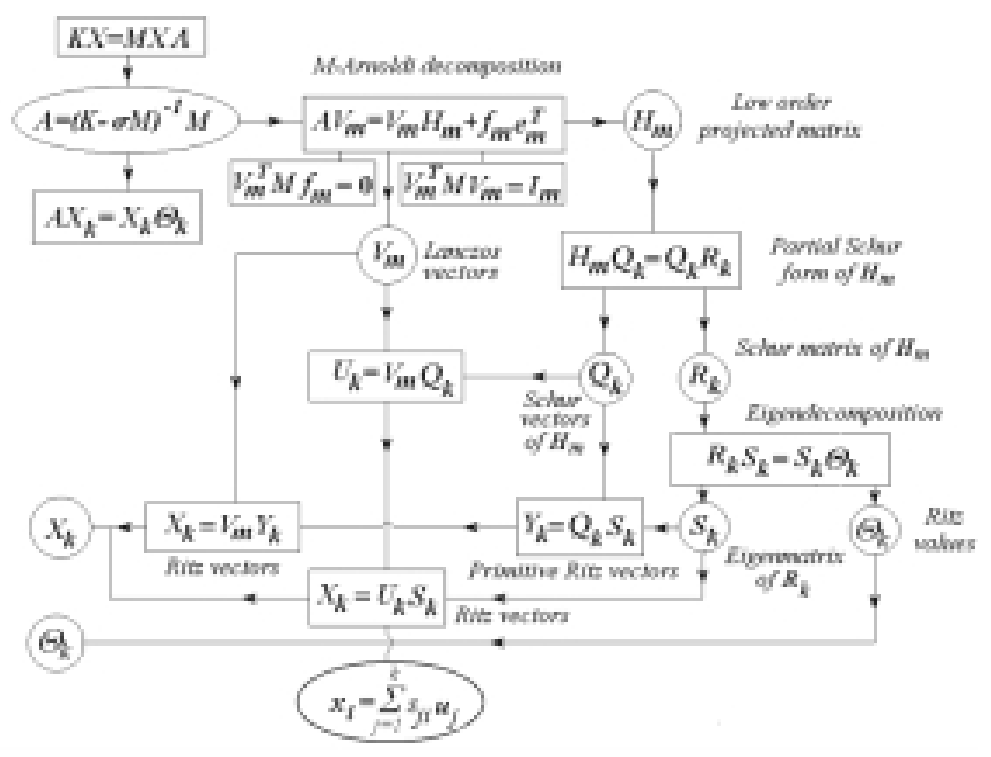

Fig. 3. Schematic of $M$-Arnoldi algorithms.

\section{Selection of active DOFs}

An important step in the pretest planning phase of modal testing is DOFs the placement of sensors. The Effective Independence (EfI) method [7] helps ranking a set of candidate active DOFs according to their contribution to the linear independence of the target modes selected in the Finite Element Model (FEM).

Let $\mathbf{X}_{r}$ be the reduced matrix of target modes truncated to the $s$ candidate active DOF locations. Equation (7) becomes

$$
\mathbf{X}_{r}=\mathbf{U}_{s} \mathbf{S}_{r}
$$

where the columns of $\mathbf{U}_{s}$ are Schur vectors and $\mathbf{S}_{r}$ is upper triangular.

The orthogonal projector onto the column space of $\mathbf{X}_{r}$ is

$$
\mathbf{P}=\mathbf{X}_{r} \mathbf{X}_{r}^{+}=\mathbf{X}_{r}\left(\mathbf{X}_{r}^{T} \mathbf{X}_{r}\right)^{-1} \mathbf{X}_{r}^{T},
$$

where $^{+}$denotes the Moore-Penrose pseudoinverse.

It can be shown that it is equal to the projector onto the subspace of Schur vectors

$$
\mathbf{P}=\mathbf{U}_{s} \mathbf{U}_{s}^{+}=\mathbf{U}_{s}\left(\mathbf{U}_{s}^{T} \mathbf{U}_{s}\right)^{-1} \mathbf{U}_{s}^{T} .
$$

The diagonal element

$$
p_{j j}=\operatorname{diag}_{j}\left(\mathbf{U}_{s} \mathbf{U}_{s}^{+}\right)
$$

is the Effective Independence value corresponding to the $j$-th sensor. It represents the fractional contribution of the $j$-th DOF to the rank of $\mathbf{P}$, and hence to the independence of the Schur vectors. The known strategy [7] is to sort the elements $p_{j j}$ serially, based on magnitude. At each step, the smallest element $p_{j j}$ is eliminated from the candidate set. The corresponding row is discarded from the matrix $\mathbf{U}_{s}$, recomputing the matrix $\mathbf{P}$ with the deflated matrix $\mathbf{U}_{s}$ until the desired number of sensors is attained.

Based on Eq. (10), Lanczos vectors can be used in Eq. (17) instead of Schur vectors to locate the active DOFs, using the projector

$$
\mathbf{P}=\mathbf{V}_{s} \mathbf{V}_{s}^{+}=\mathbf{V}_{s}\left(\mathbf{V}_{s}^{T} \mathbf{V}_{s}\right)^{-1} \mathbf{V}_{s}^{T}
$$




\section{Schur TAM and Lanczos TAM}

Correlation between test and analysis mode shapes is carried out using a reduced representation of the FEM, called a test-analysis model (TAM). The TAM is defined by the mass and stiffness matrices reduced to the test DOFs. Consider the truncated modal expansion for the FEM physical displacements

$$
\mathbf{x}=\mathbf{X}_{k} \mathbf{q}
$$

where $\mathbf{q}$ is a vector of $k$ modal coordinates. Using Eq. (7), Eq. (20) becomes

$$
\mathbf{x}=\mathbf{U}_{k} \mathbf{p}
$$

where $\mathbf{p}=\mathbf{S}_{k} \mathbf{q}$ is a new set of generalized coordinates, and the columns of $\mathbf{U}_{k}$ are the Schur vectors of $\mathbf{A}$.

Equation (21) is partitioned according to the active, $a$, and the omitted, $o$, DOFs

$$
\mathbf{x}=\left\{\begin{array}{c}
\mathbf{x}_{a} \\
\mathbf{x}_{o}
\end{array}\right\}=\left\{\begin{array}{c}
\mathbf{U}_{a} \\
\mathbf{U}_{o}
\end{array}\right\} \mathbf{p}
$$

The reduction to active DOFs is defined by

$$
\mathbf{x}=\mathbf{T}_{S} \mathbf{x}_{a}
$$

in which the projection matrix of the Schur TAM is

$$
\mathbf{T}_{S}=\left[\begin{array}{c}
\mathbf{I}_{a} \\
\mathbf{U}_{o} \mathbf{U}_{a}^{+}
\end{array}\right]
$$

The reduced mass and stiffness matrices are given by

$$
\mathbf{M}_{\text {red }}=\mathbf{T}_{S}^{T} \mathbf{M} \mathbf{T}_{S}, \mathbf{K}_{\text {red }}=\mathbf{T}_{S}^{T} \mathbf{K} \mathbf{T}_{S} .
$$

so that the reduced generalized eigenproblem becomes

$$
\left(\mathbf{K}_{\mathrm{red}}-\lambda \mathbf{M}_{\mathrm{red}}\right) \mathbf{x}_{a}=0 .
$$

The Schur TAM provides almost exact frequencies and mode shapes for the target modes. The TAM eigenvectors can be expanded to the size of the full FEM and compared with the FEM eigenvectors using the MAC matrix [8].

Using Eq. (10) instead of Eq. (7) in Eq. (20), Eq. (22) becomes

$$
\mathbf{x}=\left\{\begin{array}{c}
\mathbf{x}_{a} \\
\mathbf{x}_{o}
\end{array}\right\}=\left\{\begin{array}{c}
\mathbf{V}_{a} \\
\mathbf{V}_{o}
\end{array}\right\} \mathbf{p}
$$

where $\mathbf{p}=\mathbf{Y}_{k} \mathbf{q}$. The reduction to active DOFs is defined by

$$
\mathbf{x}=\mathbf{T}_{L} \mathbf{x}_{a}
$$

in which the projection matrix of the Lanczos TAM is

$$
\mathbf{T}_{L}=\left[\begin{array}{c}
\mathbf{I}_{a} \\
\mathbf{V}_{o} \mathbf{V}_{a}^{+}
\end{array}\right]
$$

and

$$
\mathbf{M}_{\text {red }}=\mathbf{T}_{L}^{T} \mathbf{M} \mathbf{T}_{L}, \mathbf{K}_{\text {red }}=\mathbf{T}_{L}^{T} \mathbf{K} \mathbf{T}_{L} .
$$

The Lanczos TAM is less accurate than the Modal TAM [8] but more robust. Its performance depends on the selection of the starting vector and the size of the Lanczos basis.

Both Schur and Lanczos TAMs can be constructed without solving the full eigenvalue problem. 
Table 1

Eigenfrequencies from the full FEM and the Lanczos TAM

\begin{tabular}{cccc}
\hline $\mathrm{Nr}$ & $\begin{array}{c}\text { Full FEM } \\
\text { Eigenfrequency, Hz }\end{array}$ & \multicolumn{2}{c}{ Lanczos TAM } \\
\cline { 3 - 4 } & 45.150 & 45.150 & 0 \\
2 & 79.047 & 79.047 & 0 \\
3 & 227.183 & 227.183 & 0 \\
4 & 249.673 & 249.673 & 0 \\
5 & 363.558 & 363.558 & 0 \\
6 & 437.875 & 438.084 & 0.05 \\
7 & 446.071 & 446.138 & 0.02 \\
8 & 469.421 & 494.252 & 5.29 \\
\hline
\end{tabular}

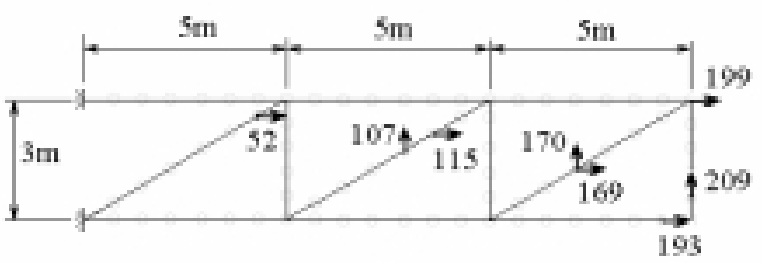

$a$

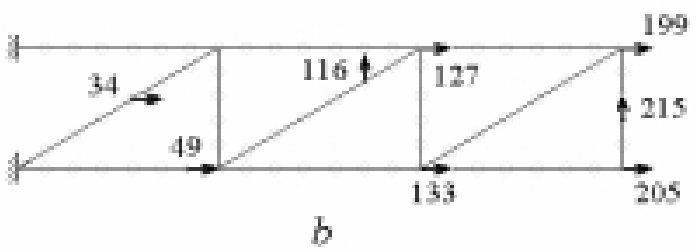

Fig. 4. Selection of eight active DOFs using: a) Schur vectors, b) Lanczos vectors.

\section{Numerical simulation}

An application is presented for the planar frame structure shown in Fig. 4, used in the third GARTEUR updating exercise, with the following data: $\rho=2800 \mathrm{~kg} / \mathrm{m}^{3}, E=0.75 \cdot 10^{11} \mathrm{~Pa}, I=0.0756 \mathrm{~m}^{4}, A_{\text {vert }}=0.006 \mathrm{~m}^{2}$, $A_{\text {horiz }}=0.004 \mathrm{~m}^{2}, A_{\text {diag }}=0.003 \mathrm{~m}^{2}$ [5]. The structure is constrained to vibrate only in its own plane.

It is modelled with 78 Bernoulli-Euler beam elements, with consistent mass matrices. The model consists of 72 free nodes resulting in 144 translational and 72 rotational DOFs. The horizontal beams, of $5 \mathrm{~m}$ length, and the diagonal beams are modelled with 7 elements each. The vertical beams, of $3 \mathrm{~m}$ length, are modelled with 5 elements each.

The first eight natural frequencies of the system from Fig. 4 are given in Table 1. The FEM values, computed for the full eigenvalue problem $(n=216)$ have been determined using the JDQR algorithm and its MATLAB implementation jdqr.m [2].

The Schur TAM has been constructed using 6 target modes and 8 active DOFs, shown in Fig. 4a, selected by the EfI technique using only translational DOFs and based on the orthogonal projector onto the column space of Schur vectors Eq. (17).

The first eight natural frequencies calculated using the Schur TAM are exactly the same as those computed using the full FEM. The expanded Schur vectors are also similar to the modal vectors. Figure 5 illustrates the first six eigenmodes. For the first five modes, the diagonal entries of the MAC matrix have unit values, and for the other three eigenvectors they are larger than 0.997.

A Lanczos TAM has been constructed using the active DOFs shown in Fig. 4b, selected by the EfI technique and based on the orthogonal projector onto the column space of Lanczos vectors Eq. (17). The natural frequencies computed using the Lanczos TAM and their relative discrepancy with respect to the FEM values are given in Table 1.

The robustness of TAMs is assessed with a global figure of merit, based on the Normalized Cross-Orthogonality (NCO) matrix [5,8], computed based on the consistent mass matrix. The NCOrms error is calculated as 


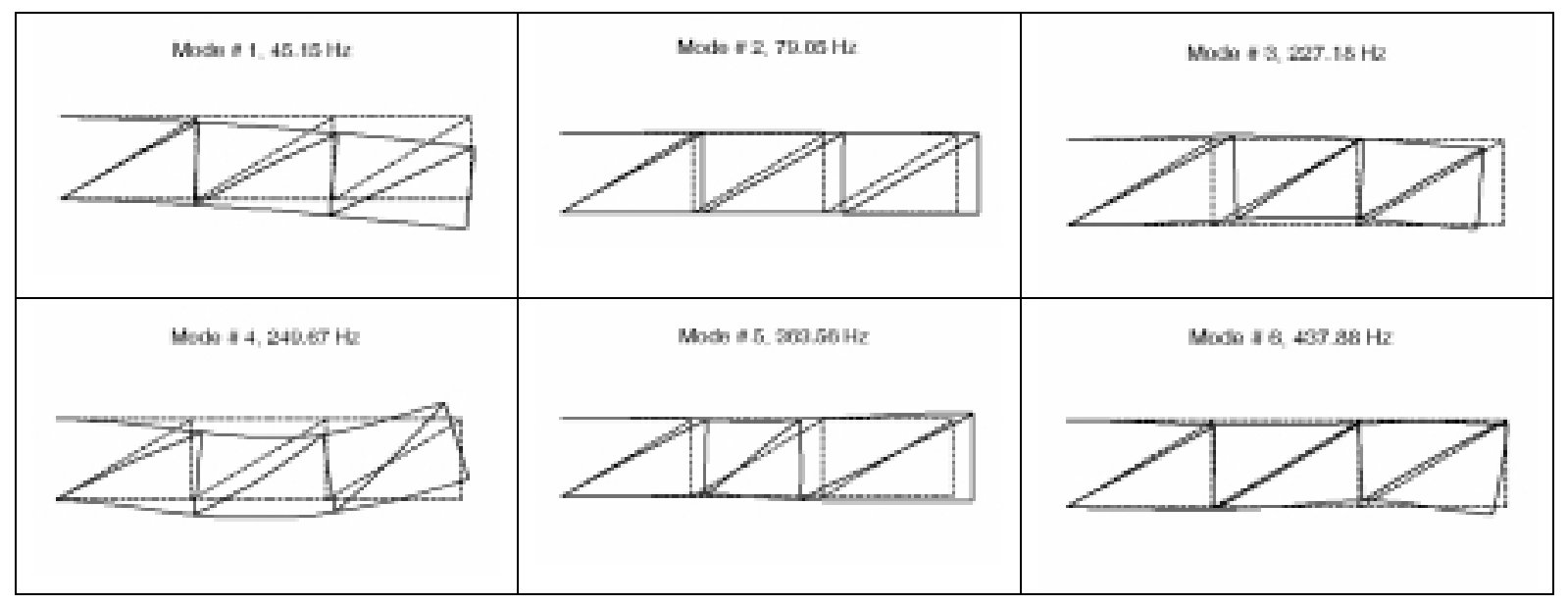

Fig. 5. Eigenmodes of the system from Fig. 4.

$r m s 1=\operatorname{rms}(\mathbf{N C O}-\operatorname{diag}(\mathbf{N C O}))$.

It is a measure of the lack of orthogonality between two sets of vectors. We obtained $r m s 1=0.136$ for the Schur TAM and $r m s 1=0.084$ for the Lanczos TAM. The low value indicates the good performance of the Lanczos TAM. The Schur TAM preserves the exact reduction features of the Modal TAM but has intrinsic reduced robustness, being based on a limited number of Schur vectors which resemble the target eigenmodes.

\section{Concluding remarks}

This paper presented the use of Schur and Lanczos vectors in the solution of large eigenvalue problems which arise in the dynamic analysis of large or geometrically complicated structural models [9]. Selection of active degrees of freedom (sensor location) is done using Lanczos vectors, instead of eigenvectors, in the Effective Independence method. New test-analysis models are constructed using a five step procedure: a) operating matrix preparation; b) Lanczos subspace generation; c) Rayleigh-Ritz eigenvalue approximation; d) selection of sensor locations using the projector onto the subspace of Lanczos vectors , and e) construction of the TAM. For moderate eigenvalue problems, the Schur decomposition of the shift-and-inverse transform matrix is used for sensor location and TAM construction without solving the eigenproblem.

\section{References}

[1] G.W. Stewart, Matrix Algorithms, Volume II: "Eigensystems", SIAM, Philadelphia, 2001.

[2] Z. Bai, J. Demmel, J. Dongarra, A. Ruhe and H. van der Vorst, Templates for the Solution of Algebraic Eigenvalue Problems: A Practical Guide, SIAM, Philadelphia, 2000.

[3] R.J. Radke, A Matlab Implementation of the Implicitly Restarted Arnoldi Method for Solving Large-Scale Eigenvalue Problems, M.A. Thesis, Rice University, Houston, Texas, 1996.

[4] D.C. Sorensen, Implicit Application of Polynomial Filters in a k-Step Arnoldi Method, SIAM J. Matrix Analysis and Applications 13 (1992), 357-385.

[5] M. Radeş and D.J. Ewins, Some Applications of Arnoldi Vectors in Modal Testing, Proceedings of IMAC-XXI Conference on Structural Dynamics, Kissimmee, Florida, Feb 2003, Paper S11P06.

[6] M. Radeş, Test-Analysis Correlation Using Schur Vectors, Proceedings of ISMA2004 Int. Conference on Noise and Vibration Engineering, Leuven, Belgium, Sept 2004, 1917-1924.

[7] D.C. Kammer, Sensor Placement for On-Orbit Modal Identification and Correlation of Large Space Structures, Journal of Guidance, Control and Dynamics 14 (1991), 251-259.

[8] M. Radeş, Comparison of Vibration Properties, in: Encyclopedia of Vibration, S.G. Braun, D.J. Ewins and S.S. Rao, eds, Academic Press, London, 2001, pp. 256-277.

[9] H.A. van der Vorst, Computational Methods for Large Eigenvalue Problems, in: Handbook of Numerical Analysis, (Vol. 8), P.G. Ciarlet and J.L. Lions, eds, North-Holland (Elsevier), Amsterdam, 2002, pp. 3-179. 

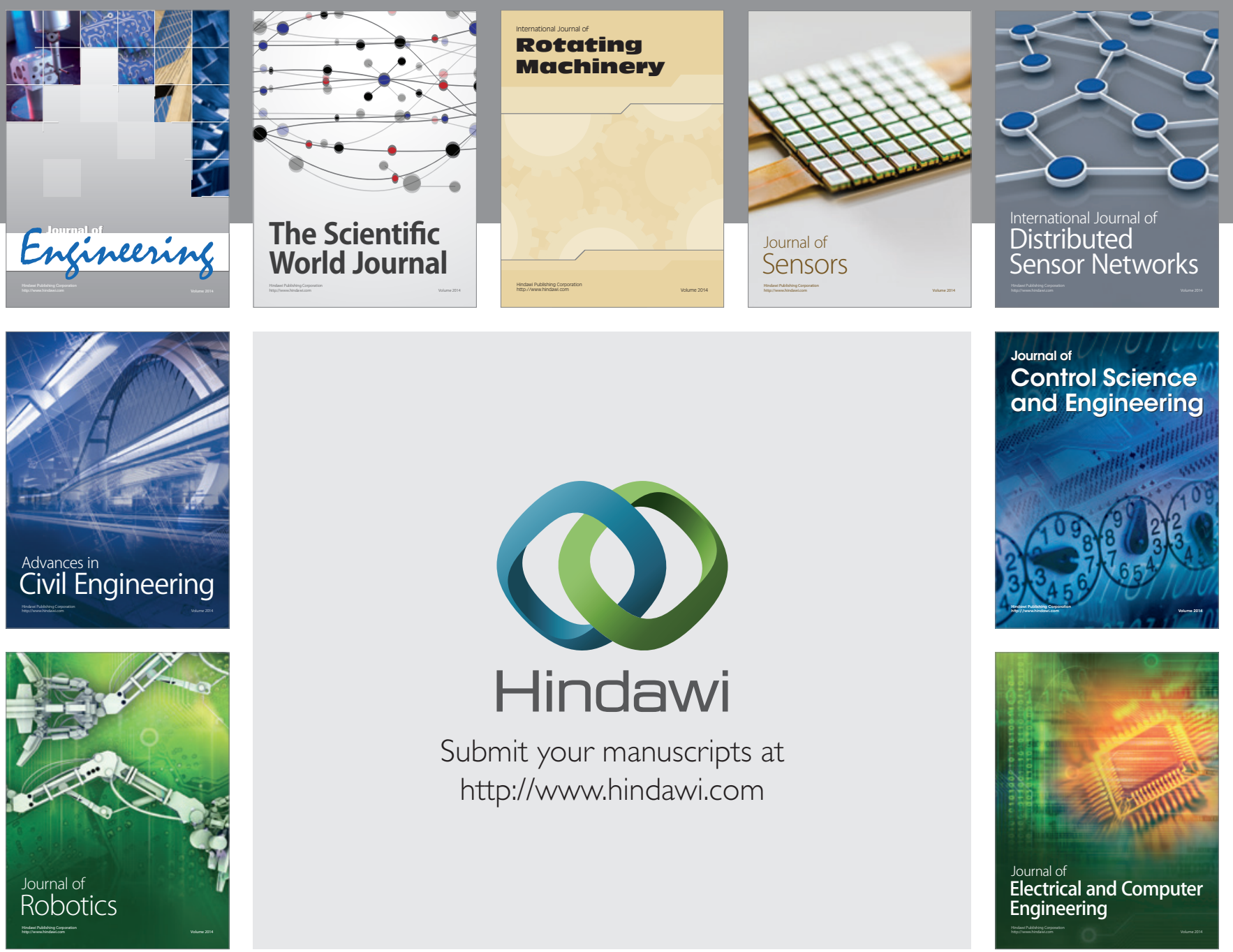

Submit your manuscripts at

http://www.hindawi.com
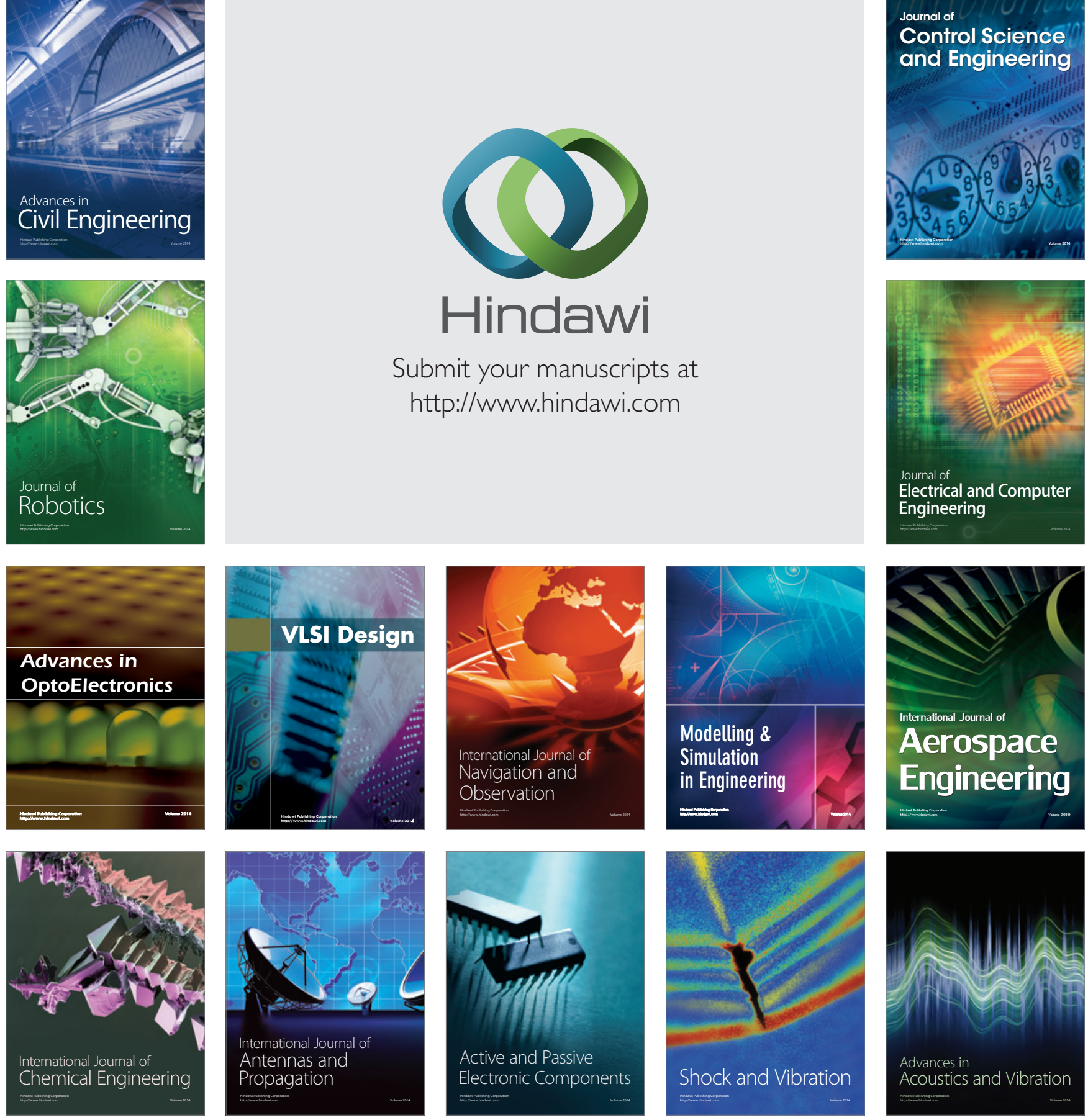